\title{
Integrated Social and Quality of Service Trust Management of Mobile Groups in Ad Hoc Networks
}

\author{
Ing-Ray Chen, Jia Guo, Fenye Bao \\ Dept. of Computer Science \\ Virginia Tech \\ E-mail: \{irchen, jiaguo, baofenye\}@vt.edu
}

\author{
Jin-Hee Cho \\ U.S. Army Research Laboratory \\ E-mail: jinhee.cho@us.army.mil
}

\begin{abstract}
We propose to combine social trust derived from social networks with quality-of-service (QoS) trust derived from communication networks to obtain a composite trust metric as a basis for evaluating trust of mobile nodes in mobile ad hoc network (MANET) environments. We develop a novel modelbased approach to identify the best protocol setting under which trust bias is minimized, that is, the peer-to-peer subjective trust as a result of executing our distributed trust management protocol is close to ground truth status over a wide range of operational and environment conditions with high resiliency to malicious attacks and misbehaving nodes.
\end{abstract}

Keywords—trust management; mobile ad hoc networks; QoS trust; social trust; trust bias minimization.

\section{INTRODUCTION}

Trust management for mobile ad hoc networks (MANETs) (see $[1,2]$ for a survey) has emerged as a new active research area as evidenced by the proliferation of trust/reputation protocols to support mobile group based applications in recent years [3-6]. In this paper we address an importance issue of trust management protocol design for MANETs: trust bias minimization despite misbehaving nodes performing trustbased attacks.

Relative to existing works [3-6] for MANET trust management cited above, this paper has two specific contributions. First, we develop a new trust management protocol (SQTrust) based on a composite social and QoS trust metric, with the goal to yield peer-to-peer subjective trust evaluation. A mobile ad hoc group very frequently comprises human operators carrying communication devices. Thus, in addition to traditional $Q o S$ trust metrics such as control packet overhead, throughput, packet dropping rate, delay, availability, convergence time to reach a steady state in trustworthiness for all participating nodes, percentage of malicious nodes, and fault tolerance, one must also consider social trust metrics including friendship, honesty, privacy, similarity, betweenness centrality and social ties for trust management. We note that prior works such as $[7,8]$ also considered social trust metrics in communication networks. Our work distinguishes itself from these prior works in that we identify the best trust aggregation parameter settings for each individual trust metric (either QoS or social) to minimize trust bias. Second, we propose a novel model-based evaluation technique for validating SQTrust based on the concept of objective trust evaluation which utilizes knowledge regarding the operational and environment conditions to yield the ground truth against which subjective trust values obtained from executing SQTrust can be compared for validation. Our analysis methodology hinges on the use of Stochastic Petri Net (SPN) mathematical modeling techniques [9-12] for describing the "actual" dynamic behaviors of nodes in MANETs in the presence of well-behaved, uncooperative and malicious nodes. With this methodology, we identify the optimal trust parameter settings under which trust bias is minimized, i.e., SQTrust is most accurate compared with global knowledge and actual node status.

\section{SQTRUST FOR MANETS}

\section{A. Trust Composition}

Taking into consideration of the proliferation of mobile devices carried by humans in social ad hoc networks, our trust metric consists of two trust types: social trust and QoS trust [1]. Social trust is evaluated through interaction experiences in social networks to account for social relationships. Among the many social trust metrics such as friendship, honesty, privacy, similarity, betweenness centrality, and social ties, we select social ties (measured by intimacy) and honesty (measured by healthiness) to measure the social trust level of a node as these social properties are considered critical for trustworthy mission execution in group settings. QoS trust is evaluated through the communication and information networks by the capability of a node to complete a mission assigned. Among the many QoS metrics such as competence, cooperation, reliability, and task performance, we select competence (measured by energy) and protocol compliance (measured by cooperativeness in protocol execution) to measure the QoS trust level of a node since competence and cooperation are considered the most critical QoS trust properties for mission execution in group settings. Quantitatively, let a node's trust level toward another node be a real number in the range of $[0$, $1]$, with 1 indicating complete trust, 0.5 ignorance, and 0 complete distrust.

The rationale of selecting these social and QoS trust metrics is given as follows. The intimacy component (for measuring social ties) has a lot to do with if two nodes have a lot of direct or indirect interaction experiences with each other, for example, for packet routing and forwarding. The healthiness component (for measuring honesty) is essentially a belief of whether a node is malicious or not. We relate it to the probability that a node is not compromised. The energy component refers to the residual energy of a node, and for a MANET environment, energy is directly related to the survivability capability of a node to be able to execute a task completely, particularly when the current and future missions 
may require a long mission execution time. Finally, the cooperativeness component of a node is related to whether the node is cooperative in routing and forwarding packets. For mobile groups, we relate it to the trust to a node being able to faithfully follow the prescribed protocol such as relaying and responding to group communication packets.

We assert that a node can have fairly accurate trust assessments toward its 1-hop neighbors utilizing monitoring, overhearing and snooping techniques. For example, a node can monitor interaction experiences with a target node within radio range, and can overhear the transmission power and packet forwarding activities performed by the target node over a trust evaluation window $\Delta t$ to assess the target node's energy and cooperativeness status. For a target node more than 1-hop away, a node will refer to a set of recommenders for its trust toward the remote target node.

\section{B. Trust Aggregation}

A unique feature of our trust aggregation protocol design is that we identify and apply the optimal trust parameter settings to minimize trust bias, i.e., minimizing the difference between subjective trust and objective trust. Here we define specific trust parameters used in our trust aggregation protocol design. Later in Section III we leverage a novel model-based approach developed in this paper to discover the best trust aggregation protocol settings to minimize trust bias.

Like most trust aggregation protocols for MANETs [1], we consider both direct trust and indirect trust. That is, node $i$ evaluates node $j$ at time $t$ by direct observations and indirect recommendations. Direct observations are direct evidences collected by node $i$ toward node $j$ over the time interval $[t-d \Delta t, t]$ when node $i$ and node $j$ are 1-hop neighbors at time $t$. Here $\Delta t$ is the trust update interval and $d$ is a design parameter specifying the extent to which recent interaction experiences would contribute to intimacy. We can go back as far as $t=0$, that is, $d=t / \Delta t$, if all interaction experiences are considered equally important. Indirect recommendations are indirect evidences given to node $i$ by a subset of 1-hop neighbors selected based on two mechanisms against slandering attacks: (a) threshold-based filtering by which only trustworthy recommenders with trust higher than a minimum trust threshold are qualified as recommenders, and (b) relevance-based trust by which only recommenders with high trust in trust component $X$ are qualified as recommenders to provide recommendations about a trustee's trust component $X$.

Summarizing above, node $i$ will compute its trust toward node $j, T_{i, j}^{X}(t)$, where $X$ is a trust component by:

$$
T_{i, j}^{X}(t)=\beta_{1} T_{i, j}^{\text {direct, } x}(t)+\beta_{2} T_{i, j}^{\text {indirect, } x}(t)
$$

In Equation 1, $\beta_{1}$ is a parameter to weigh node $i$ 's own information toward node $j$ at time $t$, i.e., "direct observations" or "self-information" and $\beta_{2}$ is a parameter to weigh indirect information from recommenders, i.e., "information from others," with $\beta_{1}+\beta_{2}=1$.

The direct trust part, $T_{i, j}^{\text {direct, } X}(t)$, in Equation 1 is evaluated by node $i$ at time $t$ depending on if node $i$ is a 1-hop neighbor of node $j$ at time $t$. If yes, node $i$ uses its direct observations toward node $j$ during $[t-d \Delta t, t]$ to update $T_{i, j}^{\text {direct, } X}(t)$ where $\Delta t$ is the periodic trust evaluation interval. Otherwise, it uses its old direct trust assessment at time $t-\Delta t$ multiplied by $e^{-\lambda_{d} \Delta t}$ (for exponential trust decay over time) to update $T_{i, j}^{\text {direct, } X}(t)$. Specifically, node $i$ will compute $T_{i, j}^{\text {direct, }{ }^{X}}(t)$ by:

$T_{i, j}^{\text {direct, } x}(t)=\left\{\begin{array}{l}T_{i, j}^{1-\text { hop, } x}(t) \quad \text { if } i \text { is a neighbor to } j \text { at } t \\ e^{-\lambda_{d} \Delta t} \times T_{i, j}^{\text {direct, } X}(t-\Delta t) \quad \text { otherwise }\end{array}\right.$

Here we note that $T_{i, j}^{\text {direct, } X}(t)$ replaces $T_{i, j}^{\text {direct, } X}(t-$ $\Delta t$ ) after the computation. So there won't be a storage overflow problem. To account for trust decay over time, we adopt an exponential time decay factor, $e^{-\lambda_{d} \Delta t}$, to satisfy the desirable property that trust decay must be invariable to the trust update frequency. Depending on the trust evaluation interval $\Delta t$, we can fine tune the value of $\lambda_{d}$ to test the effect of trust decay over time. The notation $T_{i, j}^{1-h o p, x}(t)$ here refers to the new "direct" trust assessment at time $t$. We adopt the Bayesian trust/reputation model [13] with Beta (A, B) distribution such that $\mathrm{A} /(\mathrm{A}+\mathrm{B})$ is the estimated direct trust with $\mathrm{A}$ as the number of positive service experiences and $\mathrm{B}$ as the number of negative service experiences.

The indirect trust part, $T_{i, j}^{\text {indirect, }} X(t)$ in Equation 1 is evaluated by node $i$ at time $t$ by taking in recommendations from a subset of 1-hop neighbors selected following the threshold-based filtering and relevance-based trust selection criteria. Specifically, node $i$ will compute $T_{i, j}^{\text {indirect, } X}(t)$ by:

$$
T_{i, j}^{\text {indirect, } X}(t)= \begin{cases}\frac{\sum_{m \in V}\left(T_{i, m}^{X}(t) \times T_{m, j}^{X}(t)\right)}{n_{r}} & \text { if } n_{r}>0 \\ e^{-\lambda_{d} \Delta t} \times T_{i, j}^{\text {indirect }, X}(t-\Delta t) & \text { if } n_{r}=0\end{cases}
$$

In Equation 3, $m$ is a recommender and $V$ is a set of $n_{r}$ recommenders chosen by node $i$ from its 1-hop neighbors which satisfy the threshold-based filtering and relevancebased trust selection criteria. That is, these are the recommenders for which node $i$ 's $T_{i, m}^{X}(t)$ in trust component $X$ is higher than a minimum threshold denoted by $T_{t}^{X}$. Here we note that when a recommender node, say, node $m$, provides its recommendation to node $i$ for evaluating node $j$ in trust component $X$, node $i$ 's trust in node $m$ is also taken into consideration as reflected in the product term on the right hand side of Equation 3. This accounts for trust decay over space. If $n_{r}=0$ then $T_{i, j}^{\text {indirect, } X}(t)=e^{-\lambda_{d} \Delta t} \times T_{i, j}^{\text {indirect, } X}(t-\Delta t)$ to account for trust decay over time.

\section{Trust Formation}

While many trust formation models exist [1], we adopt the importance-weighted-sum model with which trust is an importance-weighted sum of social trust and QoS trust. It encompasses more-social-trust, more-QoS-trust, social-trustonly, and QoS-trust-only in trust formation. It is particularly applicable to missions where context information is available about the importance of social or QoS trust properties for successful mission execution. For example, for a mission consisting of unmanned mobile nodes, the more-QoS-trust or QoS-trust-only trust formation model will be appropriate. The issue of determining optimal trust formation parameters for maximizing application performance is outside the scope of the paper and the reader is referred to [14] for more detail. The subjective trust value of node $j$ as evaluated by node $i$ at time $t$, 
denoted as $T_{i, j}(t)$, thus is computed by node $i$ as a weighted average of intimacy, healthiness, energy, and cooperativeness trust components. The assessment is done periodically in every $\Delta t$ interval. Specifically node $i$ will compute $T_{i, j}(t)$ by:

$$
T_{i, j}(t)=\sum_{X} w^{X} \times T_{i, j}^{X}(t)
$$

where $T_{i, j}^{X}(t)$ is the trust belief of node $i$ toward node $j$ in trust component $X=$ intimacy, healthiness, energy or cooperativeness and $w^{X}$ is the weight associated with $X$.

An interesting metric is the overall average "subjective" trust level of node $j$, denoted by $T_{j}^{s u b}(t)$, as evaluated by all active nodes. Once we obtain $T_{i, j}(t)$ from Equation 4, $T_{j}^{s u b}(t)$ can be computed by:

$$
T_{j}^{\text {sub }}(t)=\frac{\sum_{\text {all } i \neq j} T_{i, j}(t)}{\sum_{\text {all } i \neq j} 1}
$$

In this paper, we compare $T_{j}^{s u b}(t)$ with the "objective" trust of node $j$, denoted by $T_{j}^{o b j}(t)$, calculated based on actual, global information to see how much deviation subjective trust evaluation is from objective trust evaluation. Specifically, let $T_{j}^{o b j, X}(t)$ denote the "objective" trust of node $j$ in trust component $X$ at time $t$, which we can obtain by a mathematical model (see Section III below). Then, following Equation 4, $T_{j}^{o b j}(t)$ is calculated by:

$$
T_{j}^{o b j}(t)=\sum_{X} w^{X} \times T_{j}^{o b j, X}(t)
$$

\section{ANALYTICAL MODEL}

\section{A. Node SPN for Modeling Node Behavior}

Figure 1 shows the "node" SPN model developed for describing the lifetime behavior of a mobile node in the presence of other uncooperative and malicious nodes in a mobile group following the input operational profile. The system SPN model consists of $N$ node SPN models where $N$ is the number of nodes in the system. We utilize the node SPN model to obtain a single node's information (e.g., intimacy, healthiness, energy, and cooperativeness) and to derive its trust relationships with other nodes in the system. It also captures location information of a node as a function of time. We consider a square-shaped operational area consisting of $M \times M$ regions each with the width and height equal to radio radius $R$. The node mobility model is specified as part of the operational profile.

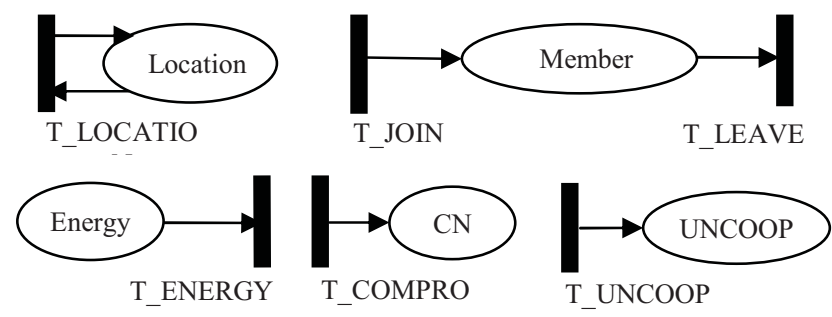

Figure 1: Node SPN Model.

The reason of using node SPN models is to yield a probability model (a semi-Markov chain) to model the stochastic behavior of nodes in the system, given the system's anticipated operational profile as input. The theoretical analysis yields objective trust based on ground truth of node status, against which subjective trust as a result of executing our proposed trust protocol is compared. This provides the theoretical foundation that subjective trust (from protocol execution) is accurate compared with ground truth. The underlying semi-Markov chain has a state representation comprising "places" in the SPN model. A node's status is indicated by a 5-component state representation (Location, Member, Energy, CN, UNCOOP) with "Location" (an integer) indicating the current region the node resides, "Member" (a boolean variable) indicating if the node is a member, "Energy" (an integer) indicating the current energy level, " $\mathrm{CN}$ " (a boolean variable) indicating if the node is compromised, and "UNCOOP" (a boolean variable) indicating if the node is cooperative. For example, place Location is a state component whose value is indicated by the number of "tokens" in place Location. A state transition happens in the semi-Markov chain when a move event occurs with the event occurrence time interval following a probabilistic time distribution such as exponential, Weibull, Pareto, and hyper-exponential distributions. This is modeled by a "transition" with the corresponding firing time in the SPN model. Below we explain how we construct the node SPN model.

Location: Transition T_LOCATION is triggered when the node moves to another region from its current location with the rate calculated as $S_{\text {init }} / R$ (i.e., the node's mobility rate) based on an initial speed $\left(S_{\text {init }}\right)$ and wireless radio range $(R)$. Depending on the location a node moves into, the number of tokens in place Location is adjusted. Suppose that nodes move randomly. Then a node randomly moves to one of four locations in four directions (i.e., north, west, south, and east) in accordance with its mobility rate. The underlying semi-Markov model of the node SPN model when solved gives the probability that a node is at a particular location at time $t$, e.g., the probability that node $i$ is located in region $j$ at time $t$. This information along with the location information of other nodes at time $t$ provides global information if two nodes are 1-hop neighbors at time $t$.

Intimacy: Intimacy trust is an aggregation of direct interaction experience $\left(T_{i, j}\right.$ direct,intimacy $\left.(t)\right)$ and indirect interaction experience $\left(T_{i, j}^{\text {indirect,intimacy }}(t)\right)$. Out of these two, only new

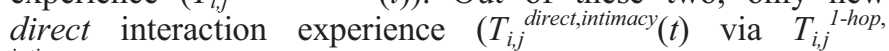
intimacy $(t))$ is calculated based on if two nodes are 1-hop neighbors interacting with each other via packet forwarding and routing. Since the node SPN model gives us the probability that a node is in a particular location at time $t$, we can objectively compute direct interaction experience $T_{i, j}^{1-h o p \text {, intimacy }}(t)$ (see Equation 2 ) based on the probability of nodes $i$ and $j$ are in the same location at time $t$ from the output of the two SPN models associated with nodes $i$ and $j$.

Energy: Place Energy represents the current energy level of a node. An initial energy level of each node is assigned differently to reflect node heterogeneity. We randomly generate a number between 12 to 24 hours based on uniform distribution, representing a node's initial energy level $E_{\text {init }}$. Then we put a number of tokens in place Energy corresponding to this initial energy level. A token is taken out when transition T_ENERGY fires. The transition rate of $T_{-}$ENERGY is adjusted on the fly based on a node's state: it is lower when a 
node becomes uncooperative to save energy and is higher when the node becomes compromised so that it performs attacks more and consumes energy more. Therefore, depending on the node's status, its energy consumption is dynamically changed.

Healthiness: A node is compromised when transition T_COMPRO fires. The rate to transition T_COMPRO is $\lambda_{\text {com }}$ as the node compromising rate (or the capture rate) reflecting the hostility of the application. If the node is compromised, a token goes to $C N$, meaning that the node is already compromised and may perform good-mouthing and badmouthing attacks as a recommender by good-mouthing a bad node with a high trust recommendation and bad-mouthing a good node with a low trust recommendation.

Cooperativeness: Place $U N C O O P$ represents whether a node is cooperative or not. If a node becomes uncooperative, a token goes to UNCOOP by triggering T_UNCOOP. The rate to transition T_UNCOOP is modeled as a function of its remaining energy, the mission difficulty, and the neighborhood uncooperativeness degree as follows:

$$
\text { rate }\left(T_{-} U N C O O P\right)=\frac{f_{e}\left(E_{\text {remain }}\right) f_{m}\left(M_{\text {difficulty }}\right) f_{s}\left(S_{\text {degree }}\right)}{T_{g c}}
$$

where $E_{\text {remain }}$ represents the node's current energy level as given in place Energy, $M_{\text {difficulty }}$ is the difficulty level of the given mission, $S_{\text {degree }}$ is the degree of uncooperativeness computed based on the ratio of uncooperative nodes to cooperative nodes among 1-hop neighbors and $T_{g c}$ is the group communication interval over which a node may decide to become uncooperative in protocol execution and drop packets. The form $f(x)=\alpha x^{-\varepsilon}$ follows the demand-pricing relationship in Economics [15-17] to model the effect of its argument $x$ on the uncooperative behavior, including:

- $f_{e}\left(E_{\text {remain }}\right)$ : If a node has a lower level of energy, it is less likely to be cooperative. This is to consider a node's individual utility in resource-constrained environments.

- $f_{m}\left(M_{\text {difficulty }}\right)$ : If a node is assigned to a more difficult mission, it is more likely to be cooperative to ensure successful mission execution.

- $f_{s}\left(S_{\text {degree }}\right)$ : If a node's 1-hop neighbors are not very cooperative, the node is more likely to be cooperative to complete a given mission successfully.

A compromised node is necessarily uncooperative as it won't follow the protocol execution rules. So if place $\mathrm{CN}$ contains a token, place UNCOOP will also contain a token.

\section{B. Obtaining Objective Trust for Validating SQTrust Protocol Design}

With the node behaviors modeled by a probability model (a semi-Markov chain) described above, the objective trust evaluation of node $j$ in trust component $X$, i.e., $T_{j}^{o b j, X}(t)$, can be obtained based on exact global knowledge about node $j$ as modeled by its node SPN model. To calculate each of these objective trust probabilities of node $j$, one would assign a reward of $r_{s}$ with state $s$ of the underlying semi-Markov chain of the SPN model to obtain the probability weighed average reward as:

$$
T_{j}^{o b j, X}(t)=\sum_{S \in S}\left(r_{S} * P_{S}(t)\right)
$$

for $X=$ healthiness, energy or cooperativeness, and as:

$$
T_{j}^{o b j, X}(t)=\frac{\int_{t-d \Delta t}^{t} \sum_{s \in S}\left(r_{s} * P_{S}\left(t^{\prime}\right)\right) d t^{\prime}}{d \Delta t}
$$

for $X=$ intimacy. Here $S$ indicates the set of states in the underlying semi-Markov chain of our SPN model, $r_{s}$ is the reward to be assigned to state $s$, and $P_{s}(t)$ is the probability that the system is in state $s$ at time $t$, which can be obtained by solving the underlying semi-Markov model of our SPN model utilizing known solution techniques such as SOR, Gauss Seidel, or Uniformization [12]. Table 1 summarizes specific reward assignments used to calculate $T_{j}^{o b j, X}(t)$ for $X=$ intimacy, healthiness, energy, or cooperativeness. In Table $1, E_{T}$ is the energy threshold below which the energy trust toward a node goes to 0 . Once $T_{j}^{o b j, X}(t)$ is obtained, we compute the average objective trust value of node $j, T_{j}^{o b j}(t)$, based on Equation 6. It is compared with average subjective trust of node $\mathrm{j}, T_{j}^{s u b}(t)$, defined in Equation 5 to compute

\begin{tabular}{|c|c|}
\hline $\begin{array}{c}\text { Component trust } \\
\text { probability toward node } j\end{array}$ & $r_{s}:$ reward assignment to state $s$ \\
\hline$T_{j}^{\text {obj,intimacy }}(t)$ & $\begin{array}{l}\left.1 \text { if mark( } j^{\prime} s \text { location }\right) \text { is within a } 5 \text { - } \\
\text { region neighbor area at time } t ; 0 \text { otherwise }\end{array}$ \\
\hline$T_{j}^{\text {obj,healthiness }}(t)$ & 1 if $\left(\operatorname{mark}\left(j^{\prime} s C N\right)=0\right) ; 0$ otherwise \\
\hline$T_{j}^{\text {obj,energy }}(t)$ & $\begin{array}{c}1 \text { if }\left(\text { mark }\left(j^{\prime} s \text { Energy }\right)>E_{T}\right) ; 0 \\
\text { otherwise }\end{array}$ \\
\hline$T_{j}^{\text {obj,cooperativen }}$ & $\begin{array}{c}1 \text { if }\left(\operatorname{mark}\left(j^{\prime} s \text { UNCOOP }\right)=0\right) ; 0 \\
\text { otherwise }\end{array}$ \\
\hline
\end{tabular}
trust bias obtained to validate our trust protocol design.

Table 1: Reward Assignments for Objective Trust Evaluation.

Table 2: Operational Profile for a Mobile Group Application.

\begin{tabular}{|c|c|c|c|}
\hline Parameter & Value & Parameter & Value \\
\hline \# of regions & $6 \times 6$ & $R$ & $250 \mathrm{~m}$ \\
\hline area & $1250 \mathrm{mx} 1250 \mathrm{~m}$ & $E_{\text {init }}$ & {$[12,24] \mathrm{hrs}$} \\
\hline$S_{\text {init }}$ & $(0,2] \mathrm{m} / \mathrm{sec}$. & $\varepsilon$ & 1.2 \\
\hline $1 / \lambda_{\text {com }}$ & $18 \mathrm{hrs}$ & $\alpha$ & 0.8 \\
\hline$T_{g c}$ & $120 \mathrm{sec}$. & $T_{t}^{X}$ & 0.6 \\
\hline
\end{tabular}

\section{RESULTS}

Table 2 lists the parameter set and their default values specifying the operational profile given as input for testing SQTrust for a mobile group of size of 150 nodes in MANET environments. Initially all nodes are not compromised. When a node is compromised and turns malicious, it performs goodmouthing and bad-mouthing attacks, i.e., it will provide the most positive recommendation (that is, 1) toward a bad node to facilitate collusion, and conversely the most negative recommendation (that is, 0) toward a good node to ruin the reputation of the good node. The initial trust level is set to 1 for healthiness, energy and cooperativeness because all nodes are considered trustworthy initially. The initial trust level of intimacy is set to the probability that a node is found to be in a 5-region neighbor area relative to $6 \times 6$ regions in accordance with the intimacy definition. Given this operational profile as input to the mobile group application, we aim to identify the best setting of $\beta_{1}$ : $\beta_{2}$ (with higher $\beta_{1}$ meaning more direct observations or self-information being used for subjective trust evaluation) under which trust bias is minimized, i.e., 


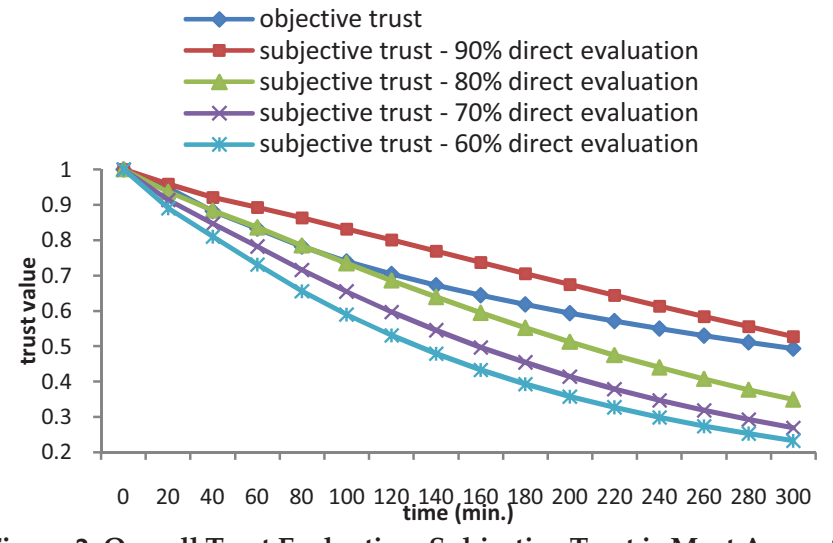

Figure 2: Overall Trust Evaluation: Subjective Trust is Most Accurate When using 85\% Direct Trust Evaluation ( $\beta 1: \beta 2=0.85: 0.15)$.

subjective trust is closest to objective trust. For trust protocol execution, we set the decay coefficient $\lambda_{d}=0.001$, and the trust evaluation interval $\Delta t=20 \mathrm{~min}$, resulting in $e^{-\lambda_{d} \Delta t}=$ 0.98 to model small trust decay over time. Also the minimum recommender threshold $T_{t}^{X}$ is set to 0.6 , the trust evaluation window size $d$ is set to 2 , and the minimum energy trust threshold $E_{T}$ is set to 0 .

Figure 2 shows the node's overall trust values obtained from subjective trust evaluation vs. objective trust evaluation, i.e., $T_{j}^{s u b}(t)$ vs. $T_{j}^{o b j}(t)$, for the equal-weight ratio case as a function of time, with $\beta_{1}$ : $\beta_{2}$ varying from $0.6: 0.4(60 \%$ direct evaluation: $40 \%$ indirect evaluation) to $0.9: 0.1$ ( $90 \%$ direct evaluation: $10 \%$ indirect evaluation). The $10 \%$ increment in $\beta_{1}$ allows us to identify the best $\beta_{1}$ : $\beta_{2}$ ratio under which subjective trust is closest to objective trust. We see that subjective trust evaluation results are closer and closer to objective trust evaluation results (and thus smaller trust bias) as we use more conservative direct observations or self-information for subjective trust evaluation. However, there is a cutoff point (at about $85 \%$ ) after which subjective trust evaluation overshoots. This implies that using too much direct observations for subjective trust evaluation could overestimate trust because there is little chance for a node to use indirect observations from trustworthy recommenders. Our analysis allows such a cutoff point to be determined to minimize trust bias, given design considerations regarding trust decay over time $\left(e^{-\lambda_{d} \Delta t}=0.98\right.$ for direct trust decay in our case study). The result is validated by ns 3 simulation (not shown due to limited space).

\section{CONCLUSION AND APPLICABILITY}

The identification of optimal protocol settings to minimize trust bias and maximize application performance is performed at static time. One way to apply the results for dynamic trust management is to build a lookup table at static time listing the optimal protocol settings discovered over a perceivable range of parameter values. Then, at runtime, upon sensing the environment conditions matching with a set of parameter values, a node can perform a simple table lookup operation augmented with extrapolation/interpolation techniques to determine and apply the optimal protocol setting to minimize trust bias in response to environment changes. In the future we plan to consider more sophisticated attacker behaviors including opportunistic, random and insidious attacks [18] to further test the resiliency of our trust protocol design.

\section{ACKNOWLEDGMENT}

This work was supported in part by the Army Research Office under Grant W911NF-12-1-0445.

\section{REFERENCES}

[1] J.H. Cho, A. Swami and I.R. Chen, "A Survey on Trust Management for Mobile Ad Hoc Networks," IEEE Communications Surveys \& Tutorials, vol. 13, no. 4, 2011, pp. 562-583.

[2] K. Govindan and P. Mohapatra, "Trust Computations and Trust Dynamics in Mobile Adhoc Networks: A Survey," IEEE Communications Surveys \& Tutorials, vol. 14, no. 2, 2012, pp. 279-298.

[3] P. B. Velloso, et al., "Trust management in mobile ad hoc networks using a scalable maturity-based model," IEEE Transactions on Network and Service Management, vol. 7, no. 3, pp. 172-185, 2010.

[4] J. Mundinger and J. Le Boudec, "Analysis of a Reputation System for Mobile Ad Hoc Networks with Liars," Performance Evaluation, vol. 65, no. 3-4, pp. 212-226, Mar. 2008.

[5] J. Luo, X. Liu, and M. Fan, "A trust model based on fuzzy recommendation for mobile ad-hoc networks," Computer Netwok, vol. 53, no. 14, pp. 2396-2407, 2009.

[6] Y. Ren and A. Boukerche, "Modeling and managing the trust for wireless and mobile ad-hoc networks," IEEE International Conference on Communications, pp. 2129-2133, Beijing, China, May 2008.

[7] S. Trifunovic, F. Legendre, and C. Anastasiades, "Social Trust in Opportunistic Networks," IEEE Conf. on Computer Communications Workshops, San Diego, CA, USA, March 2010, pp. 1-6.

[8] I. R. Chen, F. Bao, M. Chang, and J. H. Cho, "Trust Management for Encounter-Based Routing in Delay Tolerant Networks," IEEE Global Communications Conference, Miami, Florida, USA, Dec. 2010, pp. 1-6.

[9] I.R. Chen, T.M. Chen, and C. Lee, "Performance evaluation of forwarding strategies for location management in mobile networks," The Computer Journal, vol. 41, no. 4, pp. 243-253, 1998.

[10] I.R. Chen, D.C. Wang, "Analysis of replicated data with repair dependency," The Computer Journal, vol. 39, no. 9, pp. 767-779, 1996.

[11] I.R. Chen, D.C. Wang, "Analyzing Dynamic Voting using Petri Nets," 15th IEEE Symposium on Reliable Distributed Systems, Niagara Falls, Canada, 1996, pp. 44-53.

[12] K.S. Trivedi, Stochastic Petri Net Package, Duke University, 1999.

[13] A. Josang and R. Ismail, "The Beta Reputation System," 15th Conference on Electronic Commerce, Bled, Slovenia, June 17-19, 2002, pp. 1-14.

[14] F. Bao, I.R. Chen, M. Chang, and J.H. Cho, "Hierarchical Trust Management for Wireless Sensor Networks and Its Applications to Trust-Based Routing and Intrusion Detection," IEEE Transactions on Network and Service Management, vol. 9, no. 2, 2012, pp. 169-183.

[15] O. Yilmaz, and I.R. Chen, "Utilizing Call Admission Control for Pricing Optimization of Multiple Service Classes in Wireless Cellular Networks," Computer Communications, vol. 32, 2009, pp. 317-323.

[16] I.R. Chen and T.H. Hsi, "Performance analysis of admission control algorithms based on reward optimization for real-time multimedia servers," Performance Evaluation, vol. 33, no. 2, pp. 89-112, 1998.

[17] S.T. Cheng, C.M. Chen, and I.R. Chen, "Dynamic quota-based admission control with sub-rating in multimedia servers," Multimedia systems, vol. 8, no. 2, pp. 83-91, 2000.

[18] R. Mitchell and I. R. Chen, "Effect of Intrusion Detection and Response on Reliability of Cyber Physical Systems," IEEE Transactions on Reliability, vol. 62, no. 1, pp. 199-210, 2013. 